\title{
Optical Rectification and Second Harmonic Generation on Quasi-Realistic InAs/GaAs Quantum Dots: With Attention to Wetting Layer Effect
}

\author{
A. Khaledi-Nasab, ${ }^{1}$ M. Sabaiean, ${ }^{2}$ M. Sahrai, ${ }^{3}$ and V. Fallahi ${ }^{1}$ \\ ${ }^{1}$ Department of Laser and Optical Engineering, University of Bonab, Bonab, Iran \\ ${ }^{2}$ Department of Physics, Faculty of Science, Shahid Chamran University of Ahvaz, Ahvaz, Iran \\ ${ }^{3}$ Research Institute for Applied Physics and Astronomy, University of Tabriz, Tabriz, Iran
}

Correspondence should be addressed to A. Khaledi-Nasab; ali.khaledi1989@gmail.com

Received 17 September 2013; Accepted 10 October 2013

Academic Editors: C. Bauerle and V. Kochereshko

Copyright (C) 2013 A. Khaledi-Nasab et al. This is an open access article distributed under the Creative Commons Attribution License, which permits unrestricted use, distribution, and reproduction in any medium, provided the original work is properly cited.

In this paper, we have performed a theoretical study on nonlinear optical rectification (OR) and second harmonic generation (SHG) for three-level dome-shaped InAs/GaAs quantum dots (QDs) in the presence of wetting layer (WL). We used the compact density matrix framework and effective mass approximation to investigate the second order nonlinear phenomena on InAs/GaAs QD. It is demonstrated that second harmonic generation (SHG), optical rectification (OR), and their mutual absorption and refractive index changes are quite sensitive to the size of QDs. The size variations have profound irregular behavior owing to distribution of envelope function on WL and QD simultaneously. Moreover it is found that $R=13 \mathrm{~nm}$ is a critical radius where the regular variation takes place. It is shown that size variation causes blue shift until Critical radius $(R=13 \mathrm{~nm})$ and after that, increasing the QD size lead to redshift in second order phenomena.

\section{Introduction}

In the last few years the nonlinear optical properties of intersubband transitions in semiconducting materials have attracted remarkable attention due to the potential application in electronics and optoelectronics devices [1-13]. Among the semiconductor materials, zero-dimensional quantum dots (QDs) are very interesting; tunable features of QDs are one of the main reasons for this increasing performance of semiconductor QDs. The rapid advances in nanotechnology techniques such as molecular beam epitaxy (MBE) $[14,15]$, metal-organic chemical vapor deposition [16], and StranskiKrastanov (S-K) [17] methods have made it possible to prepare different shape and geometry of QDs [14-18]. In QDs the carriers are confined by the confinement potential, named as quantum confinement effect in all spatial directions. Confinement provides the quantization of electronic energy levels based on the size of the dots; confinement leads to significant optical nonlinearities $[11,18]$. Photons with proper energy can cause the intersublevel transitions involving large electric dipole moments $[19,20]$. Among the nonlinear optical properties, the second-order nonlinear optical property plays a crucial role because it is the simplest and the lowestorder nonlinear effect; moreover, its magnitude is usually stronger than the other optical nonlinearities [21]. The second order nonlinear optical interaction of two incident fields with optical media leads to some phenomena's like Second harmonic generation (SHG) and optical rectification (OR). To the best of our knowledge the early work on OR is dated back to 1962 by Xie and Bass et al. [21, 22] as well as the very first experimental observation of SHG reported by Franken et al. [23]. It is worthwhile to mention that the beginning of the field of nonlinear optics is taken by discovery of SHG [24]. SHG can be so efficient since nearly all incident beams at frequency $\omega$ transformed to radiation at second harmonic frequency $2 \omega$ [24]. The OR inference on semiconductor appeared in literature of 1992 under the rotating-wave and effective-mass approximation by Chuang et al. [25]. After this demonstration this sequence keeps going forward for different types of semiconductor nanostructures 
like quantum wells and quantum dots [26, 27]. The OR has been studied in several QDs systems including the cubical, disc-like, and spherical shapes [28-30]. The effect of exciton in OR for semiparabolic QDs for confined electron and hole in semiparabolic potential was studied by Baskoutas et al. [31]. Other studies show that the OR is a function of the applied electric field and confinement strength on QDs [30, 32]. In QDs the spin-orbit interaction decreases the magnitude of OR as well as the peaks which happen at lower energies but the donor impurity causes a big shift in the magnitude of the OR peaks position as reported by Chen et al. and Vaseghi et al. respectively $[33,34]$. Recently it is found that the OR in lense-shaped QDs is quite sensitive to the applied hydrostatic pressure and temperature [35]. The Polaron effect in OR and SHG in cylindrical QDs was studied by Liu et al. [36]; indeed they made a focus on the dependence of both the OR and the SHG on confinement frequency, external magnetic field, and the linear potential coefficient $V$.

The early experimental research on mid-infrared and near-field SHG in semiconductor QDs was reported by Brunhes et al. [37], Liu and Bryant [38], respectively. Indeed Brunhesa et al. $[39,40]$ showed that the resonant SHG in association with the intraband transitions of the P-type QDs grown by MBE can be achieved. Also the enhanced SHG with intersubband transition (specifically S-P transition) with conduction states of InAs/GaAs was demonstrated experimentally for the first time by Brunhesa et al. [41]. The size of the QDs influences the confinement potential and thus optical properties of low dimensional QDs face some alteration. The effect of size under different values of electric and magnetic fields on SHG for parabolic studied by Li et al. [42]; also the length-dependent SHG on cubical QDs as function of incident photon energy was presented by Shao et al. [43]. The spin-orbit interaction effects on the OR for different size of QDs were reported by Vaseghi et al. [34]. Sabeian and Khaledi-Nasab highlighted the size-dependent intersubband optical properties of dome-shaped InAs/GaAs quantum dots with wetting layer [44].

The QDs in the practical growth method always are grown on wetting layer (WL) $[45,46]$. In our study the InAs is sandwiched between two GaAs matrixes. In almost all the previous studies the second order susceptibility equations were simplified and some of the none resonant terms were removed based on some approximations. In this work we are presenting a deep understanding of the OR and SHG in a three-level dome-shaped InAs/GaAs QDs with WL using finite element method (FEM) and compact density matrix framework. The WL zone which is usually ignored in some of the preceding articles [47-63] is fully adopted as well as its effect on second order nonlinear phenomena is investigated. By taking all resonant and nonresonant terms of second order nonlinear susceptibility under consideration, the effect of all expressions is being highlighted.

\section{Theoretical Framework}

This work is concerned with the investigation of domeshaped InAs QDs. First of all we calculated the transition frequencies between the levels and envelop function in single InAs QDs by making use of finite element method (FEM) numerical solution for Schrödinger equation within the effective mass approximation. Throughout this work the InAs QDs are grown on InAs WL which is embedded in a large GaAs matrix and the GaAs cap cover the grown QDs as Figure 1(a) depicts.

By this definition we need to consider a domeshaped InAs/GaAs QD with cylindrical symmetry whose Schrödinger wave equation in one-band envelop-function formalism is given by

$$
-\frac{\hbar^{2}}{2 m^{*}} \nabla^{2} \Psi(\vec{r})+V(r) \Psi(\vec{r})=E \Psi(\vec{r}),
$$

where $m^{*}$ is the electron effective mass having two different values in QD region and in surrounding medium, $\Psi(\vec{r})$ is the electronic envelop function, and $E$ is the eigenenergy. Using the cylindrical symmetry of the presented geometry in Figure 1(a) gave the permission to separate the envelop function as

$$
\Psi(\vec{r})=\chi(r, z) \Theta(\varphi),
$$

where $r, z$, and $\varphi$ are cylindrical coordinates. Substituting (2) in (1) and dividing both sides by $\chi(r, z) \Theta(\varphi)$ give

$$
\begin{gathered}
\frac{1}{\Theta} \frac{d \Theta}{d \varphi^{2}}=-l^{2} \\
-\frac{m_{e} r^{2} h}{8 \pi^{2}} \frac{1}{\chi_{l}}\left[\frac{\partial}{\partial z}\left(\frac{1}{m_{e}} \frac{\partial \chi_{l}}{\partial z}\right)+\frac{1}{r} \frac{\partial}{\partial r}\left(\frac{r}{m_{e}} \frac{\partial \chi}{\partial r}\right)\right] \\
+m_{e} r^{2}(V-E)=-\frac{h^{2}}{8 \pi} l^{2},
\end{gathered}
$$

where $l$ is a separation constant. As the envelop function must be single-valued under $2 \pi$ rotation, the $\varphi$-part can be expressed as $\Theta(\varphi) \propto \exp (i l \varphi)$ leading to integer values of $l=$ $0, \pm 1, \pm 2, \ldots$. Equation ( 4 ) can be rearranged as

$$
\begin{gathered}
-\frac{h^{2}}{8 \pi^{2}}\left[\frac{\partial}{\partial z}\left(\frac{1}{m_{e}} \frac{\partial \chi_{l}}{\partial z}\right)+\frac{1}{r} \frac{\partial}{\partial r}\left(\frac{r}{m_{e}} \frac{\partial \chi_{l}}{\partial r}\right)\right] \\
+\left(\frac{h^{2}}{8 \pi^{2} m_{e}} \frac{1}{r^{2}}+V\right) \chi_{l}=E \chi_{l} .
\end{gathered}
$$

As it is obvious the three-dimensional Schrödinger equation is being simplified to two variable equation. To solve the Schrödinger equation we must define adequate boundary conditions. According to Figure 1(b), for the boundaries 1 (rooftop) and 2 (bottom), the condition of $\chi_{l}=0$ is considered, while for all other exterior boundaries the condition of $n \cdot\left(\nabla \chi_{l}\right)=0$ is employed where $n$ is outward unit vector. For interface boundaries of InAs QD-WL and GaAs matrixes, the condition of $n \cdot\left(\nabla \chi_{l} / m^{*}\right)_{\mathrm{GaAs}}=n \cdot\left(\nabla \chi_{l} / m^{*}\right)_{\mathrm{InAs}}$ is adopted because of the finiteness of the potential barrier. In our numerical simulations, the effective electron mass was set as $0.023 m_{e}$ for InAs and $0.069 m_{e}$ for GaAs [64], where $m_{e}$ is the free electron mass. The finite potential was taken as 0 and $0.697 \mathrm{eV}$ for InAs and GaAs, respectively. 


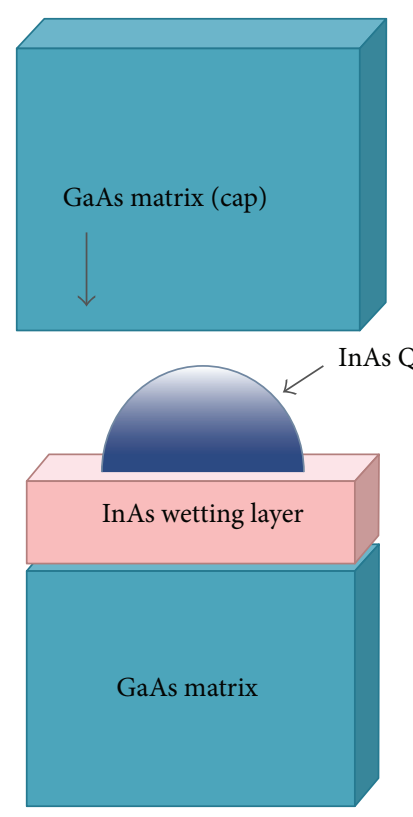

(a)

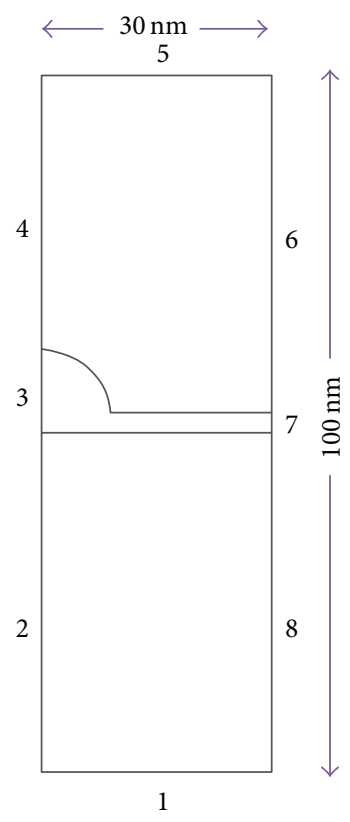

(b)

Figure 1: (a) The three-dimensional InAs QDs/WL and GaAs matrixes, (b) the two-dimensional area with numbered boundaries for simplified Schrödinger equation.

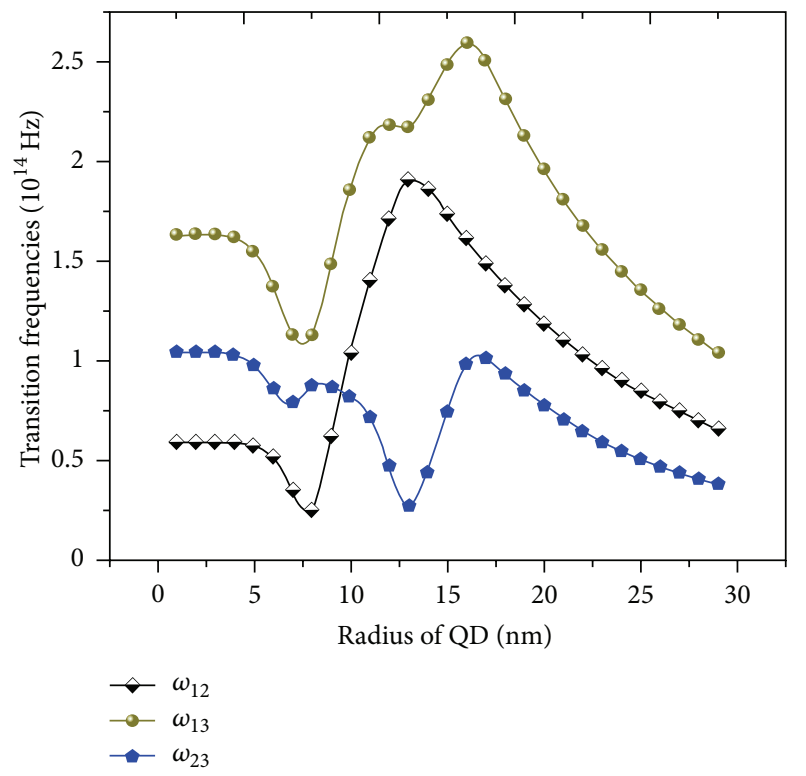

FIGURE 2: Transition frequencies for three levels in InAs/GaAs QDs versus field probe frequency. These curves show the variation of transition frequency for different radius of QDs ranging from 1 to $30 \mathrm{~nm}$. The thickness of wetting layer is chosen to be $3 \mathrm{~nm}$.

Assume that our system is excited by linear $x$-polarized monochromatic electric field propagating along $z$-direction as

$$
\widetilde{E}(z, t)=E_{0} \hat{i} e^{i(k z-\omega t)}+c . c .
$$

According to Boyd notation [24], $E=\sqrt{I / 2 n \varepsilon_{0} c}$. Based on nonlinear optics formalism [24] the refractive index changes and the absorption coefficient are given by:

$$
\begin{gathered}
\alpha=\frac{2 n_{i} \omega}{c}=\omega \alpha_{0} \operatorname{Im}\left(\chi_{\mathrm{eff}}(\omega)\right), \\
\frac{\Delta n_{r}}{n_{r}}=\frac{n_{r}-1}{n_{r}}=\frac{1}{2} \operatorname{Re}\left(\frac{\chi_{\mathrm{eff}}(\omega)}{n_{r}}\right),
\end{gathered}
$$

where $\alpha_{0}=\sqrt{\epsilon_{0}^{2} \mu / \epsilon_{R}}, \mu$ is the vacuum permeability, $\epsilon_{R}$ is the real part of permittivity, and $\epsilon_{0}$ is the vacuum permittivity. Consider $\chi_{\text {eff }}=\chi^{(1)}+\left(\chi^{(2)}(0)+\chi^{(2)}(2 \omega)\right) \widetilde{E}+\chi^{(3)} \widetilde{E}^{2}$ is effective susceptibility expressed in term of linear and nonlinear parts. In this study we are looking only for second order nonlinear susceptibility, so the absorption coefficient and refractive index changes equations will rearranged be as:

$$
\begin{gathered}
\alpha_{\left(\chi^{2}\right)}=\omega \alpha_{0} \operatorname{Im}\left(E \chi^{2}(\omega)\right), \\
\left(\frac{\Delta n_{r}}{n_{r}}\right)_{\left(\chi^{2}\right)}=\frac{1}{2} \operatorname{Re}\left(E \frac{\chi^{2}(\omega)}{n_{r}}\right) .
\end{gathered}
$$

Based on the density matrix approach [24], the second order OR and SHG relationship could be calculated. According to 


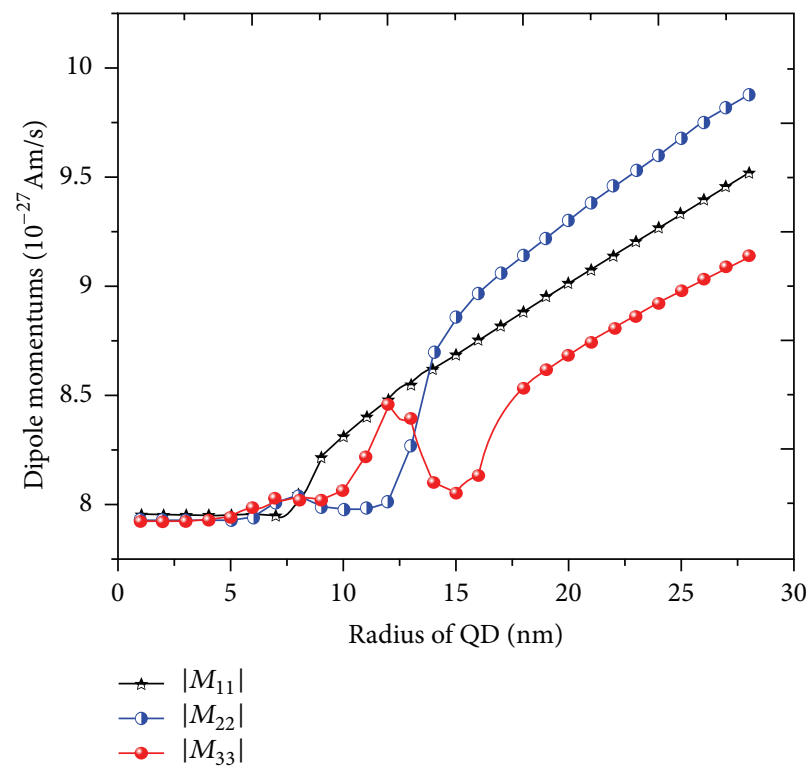

(a)

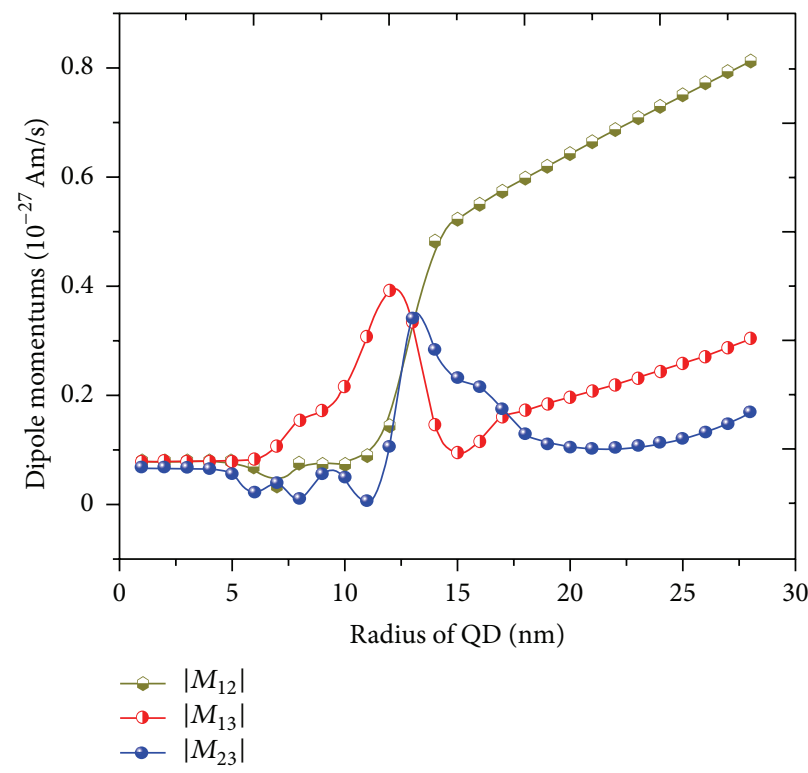

(b)

FIGURE 3: The absolute values of (a) diagonal and (b) off-diagonal elements of dipole momentum matrix versus dome radius.

this formalism, the OR and SHG for 3 levels of InAs/GaAs QD system are given by

$$
\begin{aligned}
& \chi_{(0)}^{2} \\
& =\frac{\sigma}{\varepsilon_{0} \hbar^{2}} \sum_{n, m=1}^{3} M_{1 n} M_{n m} M_{m 1} \\
& \quad \times\left\{\frac{\left(\omega_{m 1}-i \gamma_{m 1}\right)\left[\left(\omega_{n 1}+\omega_{n m}\right)+i\left(\gamma_{n m}-\gamma_{n 1}\right)\right]}{\left[\left(\omega_{m 1}-i \gamma_{m 1}\right)^{2}-\omega^{2}\right]\left(\omega_{n 1}-i \gamma_{n 1}\right)\left(\omega_{n m}+i \gamma_{n m}\right)}\right. \\
& \left.+\frac{\left(\omega_{n 1}+i \gamma_{n 1}\right)\left[\left(\omega_{m 1}+\omega_{m n}\right)+i\left(\gamma_{m 1}-\gamma_{m n}\right)\right]}{\left[\left(\omega_{n 1}+i \gamma_{n 1}\right)^{2}-\omega^{2}\right]\left(\omega_{m 1}+i \gamma_{m 1}\right)\left(\omega_{m n}-i \gamma_{m n}\right)}\right\},
\end{aligned}
$$

$$
\begin{aligned}
\chi^{2}(2 \omega)= & \frac{\sigma}{\varepsilon_{0} \hbar^{2}} \\
& \times \sum_{n, m=1}^{3} M_{1 n} M_{n m} M_{m 1} \\
& \times\left\{\frac{1}{\left[\left(\omega_{n 1}-2 \omega\right)-i \gamma_{n 1}\right]\left[\left(\omega_{m 1}-\omega\right)-i \gamma_{m 1}\right]}\right. \\
& +\frac{1}{\left[\left(\omega_{m n}-2 \omega\right)-i \gamma_{m n}\right]\left[\left(\omega_{n 1}+\omega\right)+i \gamma_{n 1}\right]} \\
& \left.+\frac{1}{\left[\left(\omega_{n m}+2 \omega\right)+i \gamma_{n m}\right]\left[\left(\omega_{m 1}-\omega\right)-i \gamma_{m 1}\right]}\right] \\
& \left.+\frac{1}{\left[\left(\omega_{m 1}+2 \omega\right)+i \gamma_{m 1}\right]\left[\left(\omega_{n 1}+\omega\right)+i \gamma_{n 1}\right]}\right\}
\end{aligned}
$$

where $\sigma$ is the density of the carriers, $M_{j i}=\left\langle\psi_{j}|-e z| \psi_{i}\right\rangle$ is the component of electric dipole moment matrix, $\omega_{j i}=\left(E_{j}-\right.$ $\left.E_{i}\right) / \hbar$ is the transition angular frequency, $\gamma_{j i}$ is the relaxation rate from level $j$ to level $i$, and $n$ and $m$ are the dummy indices, indicating the number of the levels which is 3 here.

In this case OR is purely real, so it has no contribution in absorption coefficient but SHG collaborates in both refractive index changes and absorption coefficient. It is noteworthy to indicate that we avoid opening the summation because both OR and SHG consist of almost 76 and 144 successive expression, respectively. We admit removing any nonresonant expressions since the effect of all expression will leads to more practical results.

\section{Result and Discussion}

Figure 1(b) depicts the geometry of models set as $30 \mathrm{~nm}$ and $100 \mathrm{~nm}$ for the width and height of the 2D modeling area, respectively. The height of WL has been set as $3 \mathrm{~nm}$ based on what presented in $[44,65,66]$. In the next step the radius of QDs was varied from $1 \mathrm{~nm}$ to $30 \mathrm{~nm}$. To carry out our simulations, we set the carrier density to $\sigma=3 \times 10^{22} \mathrm{~m}^{-3}$ and the damping rate as all the relaxation rates $\gamma_{j i}=5 \mathrm{ps}^{-1}[67-$ 69]. Also, the refractive index of GaAs was set as constant value $n_{r}=3.2$.

The variation of the first three transition frequency amplitudes in the sublevels of conduction band as a function of QDs size is shown in Figure 2. The first three subbands are named $s, p$, and $d$ and thus these intersubband transitions belong to these specific subbands. The diagonal and offdiagonal elements dipole momentum matrixes are illustrated in Figures 3(a) and 3(b), respectively. According to Figures 2 and 3 approximately until $R=4 \mathrm{~nm}$ the parameters take a single value with slight ignorable fluctuation. At $R=7 \mathrm{~nm}$ the 


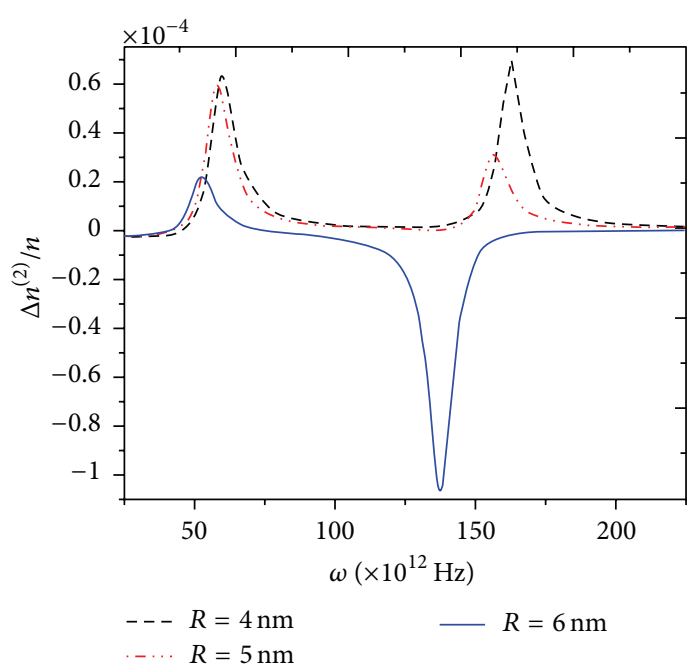

(a)

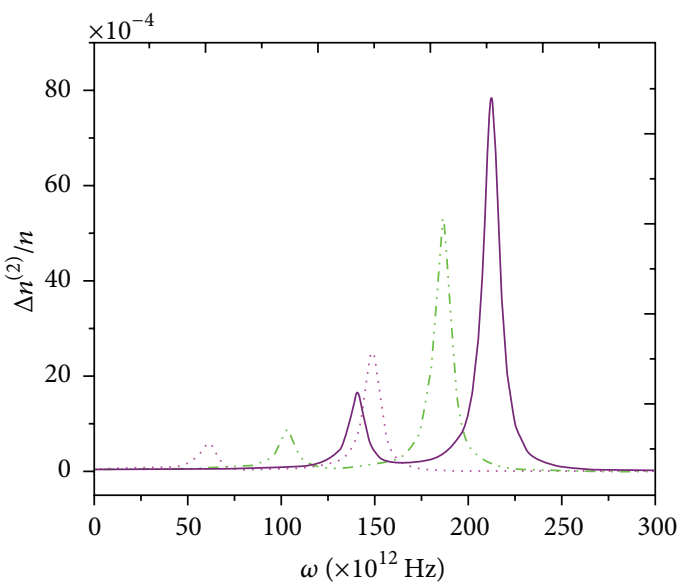

$R=9 \mathrm{~nm}$

-... $R=10 \mathrm{~nm}$

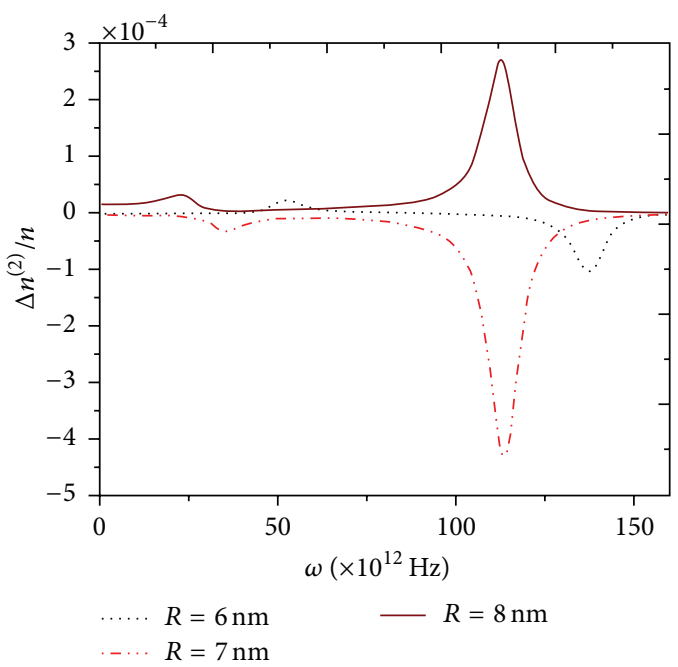

(b)

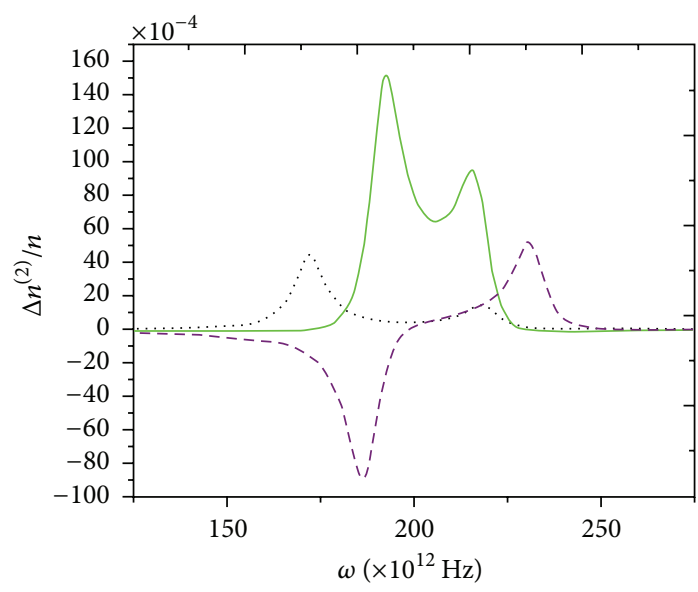

$R=12 \mathrm{~nm}$ $---R=14 \mathrm{~nm}$

(d)

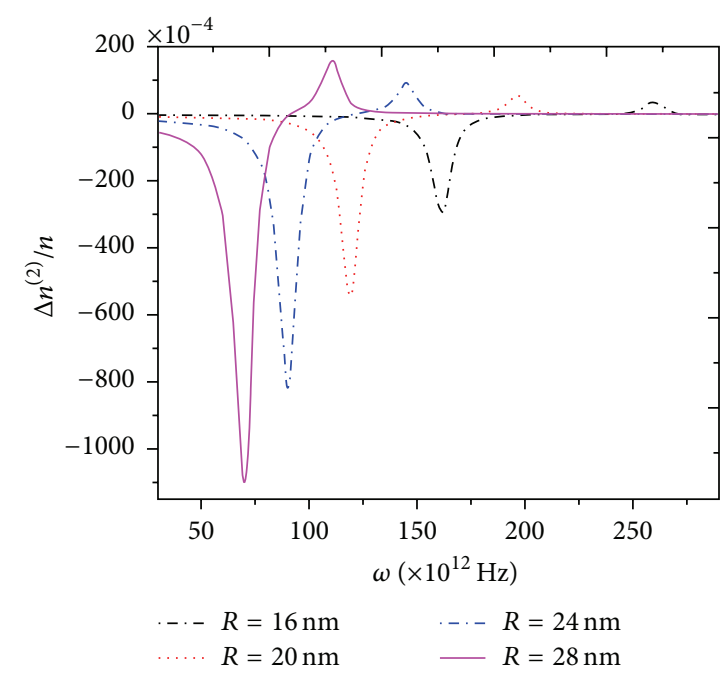

(e)

FIGURE 4: The refractive index changes due to optical rectification versus the incident light frequency for different dimensions of QDs. The amplitude of transition frequencies and dipole moments matrix is applied according to the achievement in Figures 2 and 3. 


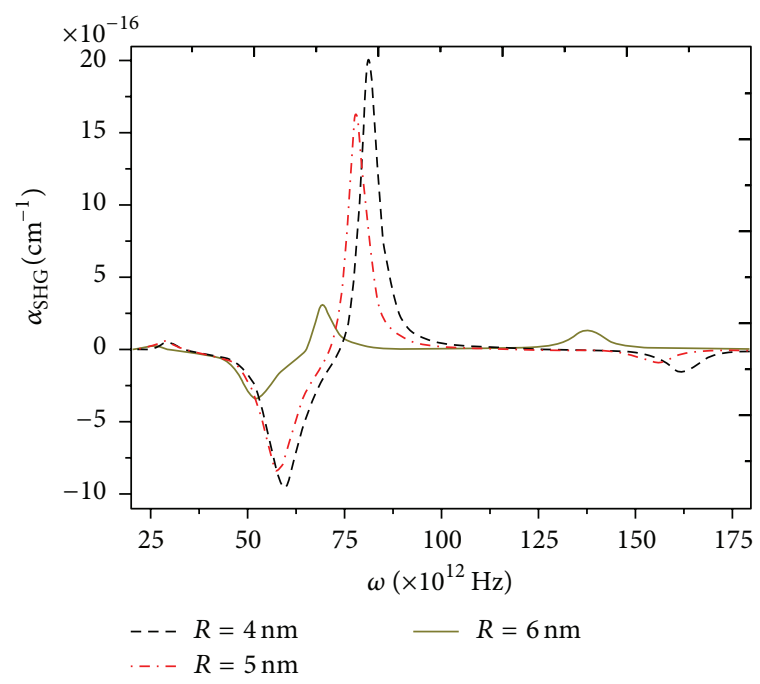

(a)

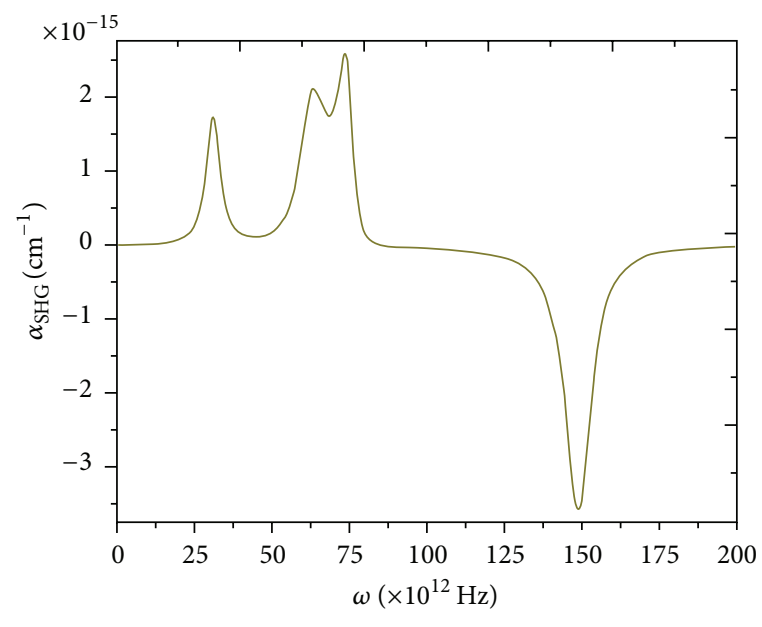

(c)

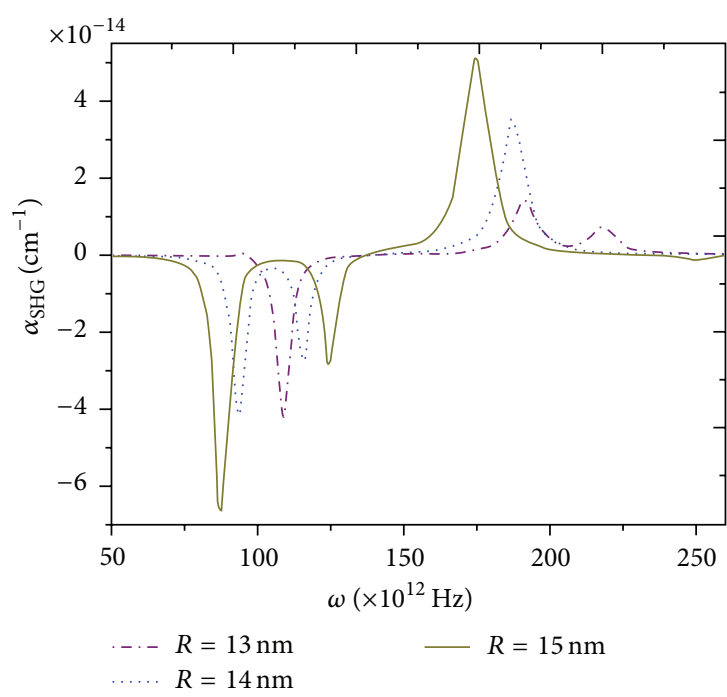

(e)

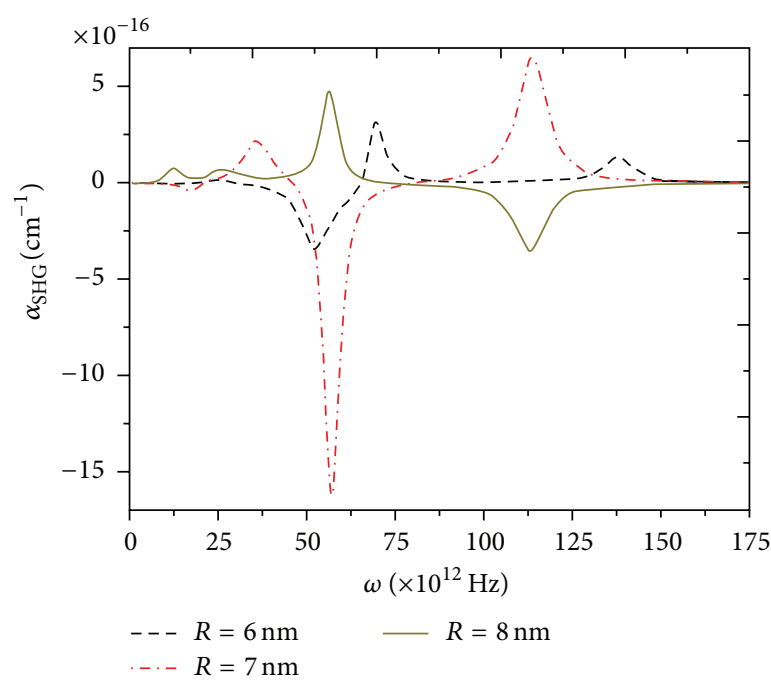

(b)

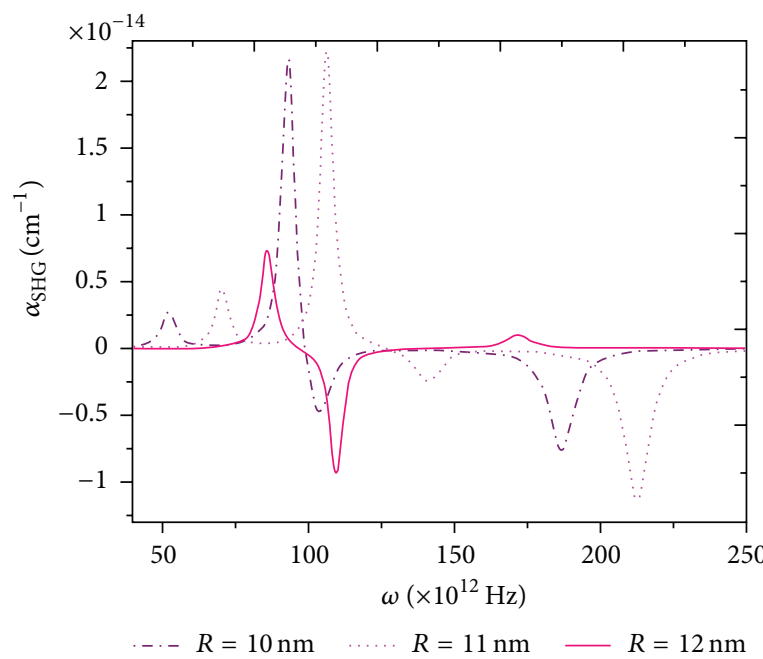

(d)

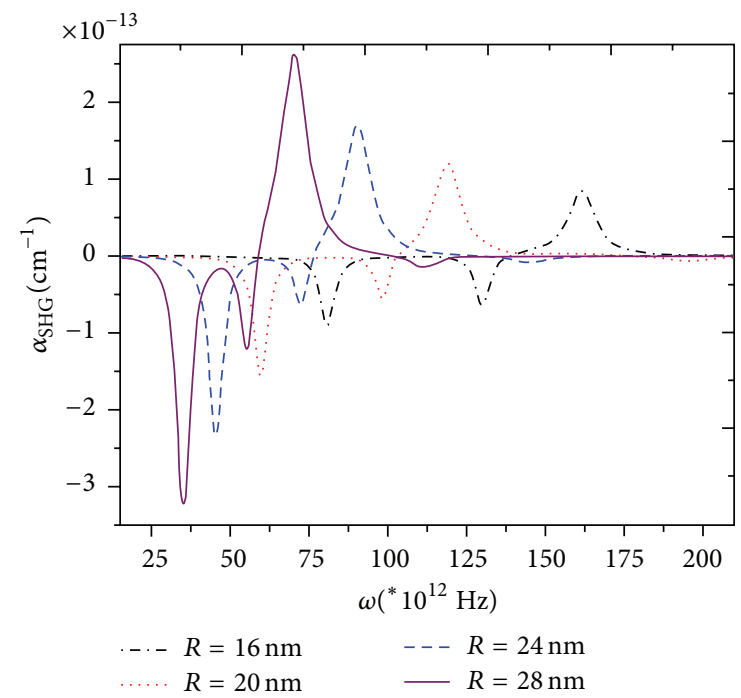

(f)

FIGURE 5: The absorption due to second harmonic generation (SHG) versus the incident light frequency for different dimensions of QDs. The physical parameters are set as in Figure 4. 

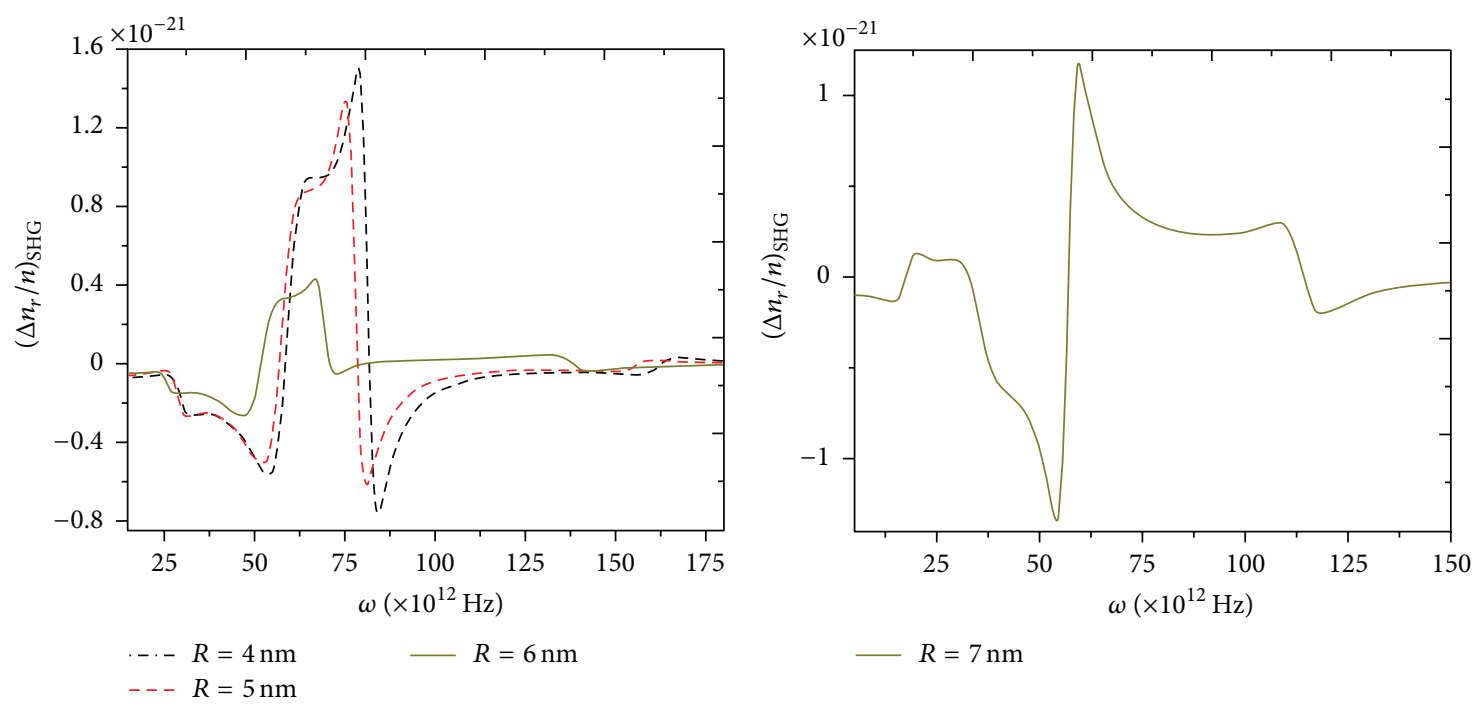

(a)

(b)
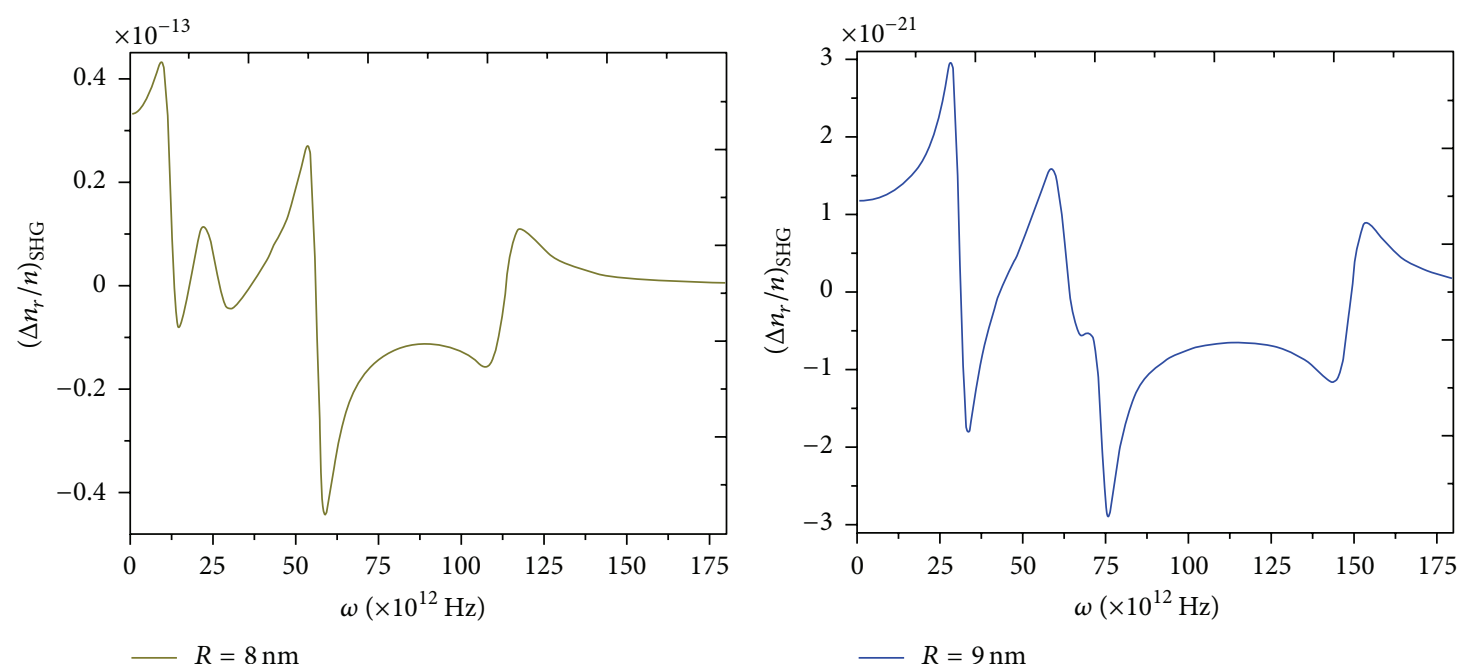

(d)

(c)
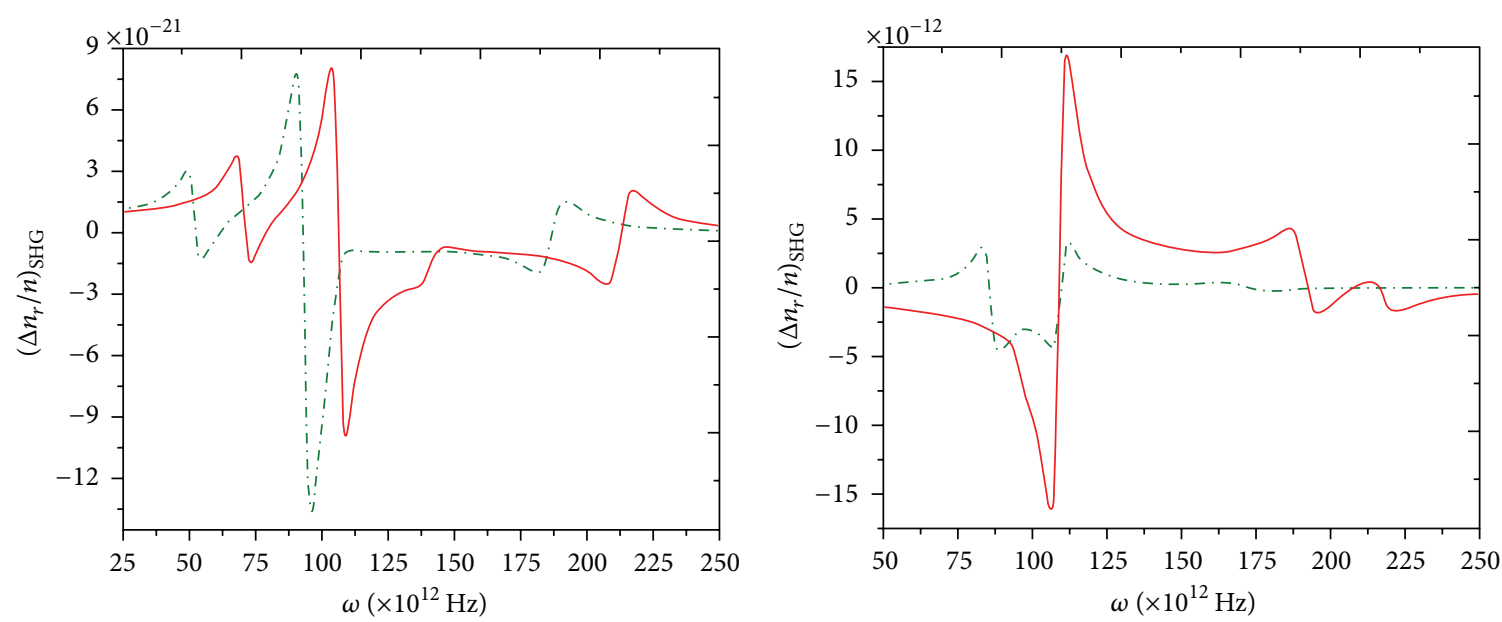

$\ldots R=10 \mathrm{~nm}$
$-R=11 \mathrm{~nm}$

-. $R=12 \mathrm{~nm}$

- $R=13 \mathrm{~nm}$

(e)

(f)

Figure 6: Continued. 


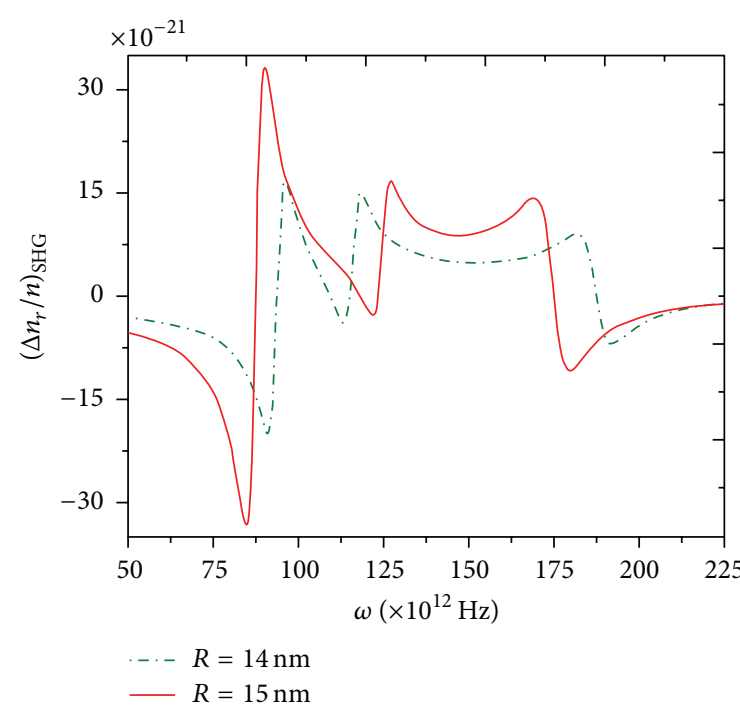

(g)

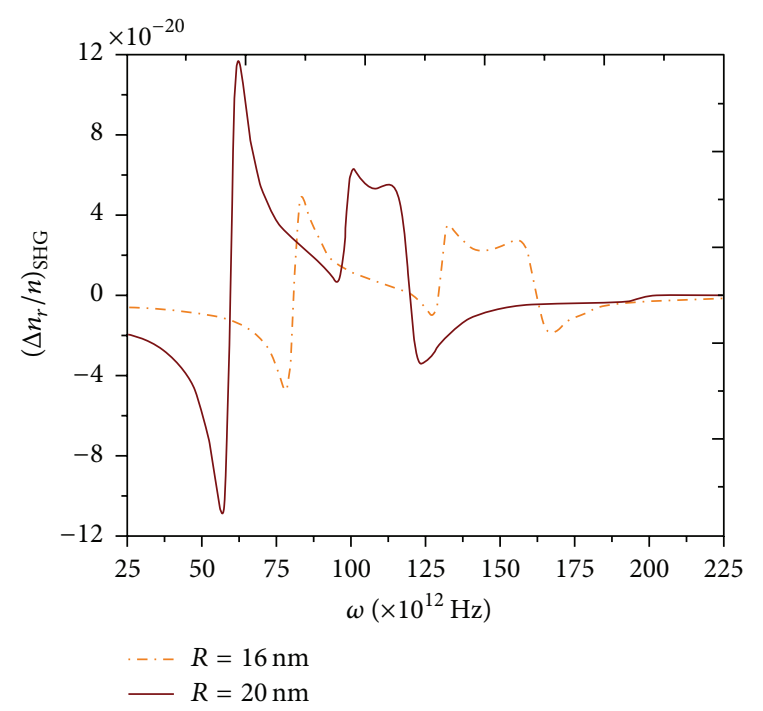

(h)

FIGURE 6: The refractive index changes due to second harmonic generation (SHG) versus the incident light frequency for different dimensions of QDs. The physical parameters are set as in Figure 4.

amplitude rises up. After $R=7 \mathrm{~nm}$ the situation becomes different in all the specific levels. The envelop function of ground state (GS, $\psi_{1}$ ) consists of single part but the envelop function on first excited state (FES, $\psi_{2}$ ) and second excited state (SES, $\psi_{3}$ ) consists of 2 and 3 individual units (lobes), respectively. When the radii of the QDs are below $5 \mathrm{~nm}$, the envelop function for all states totally holds on WL, in other words the envelop functions are localized inside the WL. At $R=5 \mathrm{~nm}$ GS envelop function and first lobes of FES and SES begin to leave the WL and enter the QD. Figure 2 reveals the dropdown for all frequencies from 5 to $8 \mathrm{~nm}$. For upper dimensions, more complicated behavior is being observed. $\omega_{12}$ and $M_{12}\left(M_{12}=\left\langle\psi_{1}|-e z| \psi_{2}\right\rangle\right)$ are related to GS and FES. $M_{12}$ also has a constant value until $R=5 \mathrm{~nm}$. When the entry of second lobe of FES begins at $R=8 \mathrm{~nm}$, simultaneously $\omega_{12}$ slope changes from negative to positive, in the same manner $M_{12}$ shows a jump on its amplitude but it still takes close values. The second lobe gets inside the dot at $R=14 \mathrm{~nm}$. After that dimension, the $\omega_{12}$ suddenly takes negative slope and for upper radii reduces with semiexponential manner. For radii larger than $15 \mathrm{~nm}$, the GS and FES become more localized inside the dot; also their its slope vanishes gradually. Moreover the $\omega_{12}$ takes linear reduction but the $M_{12}$ linear behavior increase which makes one think that the $z$ component partially controls these elements. $\omega_{13}$ and $M_{13}\left(M_{13}=\left\langle\psi_{1}|-e z| \psi_{3}\right\rangle\right)$ are multifaceted parameters since the SES has three lobes. The SES has 3 lobes which leave the WL successively. $\omega_{13}$ generally has higher absolute value than $\omega_{12}$ for every specific dimension. For $\omega_{13}$ until $R=13 \mathrm{~nm}$ everything is just like $\omega_{12}$ but after that when the third lobe of SES begins to enter the dot and Elevation occurs between $R=13 \mathrm{~nm}$ and $R=16 \mathrm{~nm}$. Afterward the expected regular sequence take place. $M_{13}$ after $R=5 \mathrm{~nm}$ until $R=8 \mathrm{~nm}$ increases but a ripple happens because entrance of first lobe in both GS and
SES ended and the Second lobe initiate its journey toward the dot. The slope is positive until $R=12 \mathrm{~nm}$. However the afterward the slop changes to negative. Since $R=16 \mathrm{~nm}$ has slight changes on the negative sequence this leads the entire behavior to have the same slope for upper radii.

$\omega_{23}$ and $M_{23}\left(M_{23}=\left\langle\psi_{2}|-e z| \psi_{3}\right\rangle\right)$ are calculated based on FES and SES. Thus we anticipate more complicated behavior. Indeed $\omega_{23}$ has smaller value than other transitions. Until $R=7 \mathrm{~nm}$ everything is like previous transitions. At $R=8 \mathrm{~nm}$ a little elevation occurs but soon after that the sequence keep moving down. The second lobes of FES and SES collaborate with each other cause this reduction until $R=13 \mathrm{~nm}$; this is due to the simultaneous movement of these lobes to the dot which intensify the decline and its slope. Afterward a promotion follows, $R=16 \mathrm{~nm}$ and in just one nanometer greater than the regular sequence shows up. $M_{23}$ has slight fluctuation until $R=8 \mathrm{~nm}$ which is not so weird because the lobes are not so synchronized. A dropdown happens at $R=10 \mathrm{~nm}$; just one nanometer beyond a significant elevation appears and keeps being like that until $R=13 \mathrm{~nm}$. For higher radii the envelop functions for all levels only become more localized and the sequence regularly just rises.

Figure 3(a) shows the intersubband dipole momentums (ISDM). These elements are discussed only for single level for transition between s, p, and d sublevels so mainly they are socalled intersubband dipole momentums. Due to Simultaneous, Confinement effect and size promotion the ISDM have greater values than those of off-diagonal elements.

As Figure 3(a) shows the absolute value of $M_{11}\left(M_{11}=\right.$ $\left.\left\langle\psi_{1}|-e z| \psi_{1}\right\rangle\right)$; when only lobe of the envelop function come inside the dot, the absolute value just keeps moving forward which looks like that the $z$ component take the lead.

The $M_{22}\left(M_{22}=\left\langle\psi_{2}|-e z| \psi_{2}\right\rangle\right)$ has a ripple on $R=8 \mathrm{~nm}$ because the first unit comes inside and the journey of the 


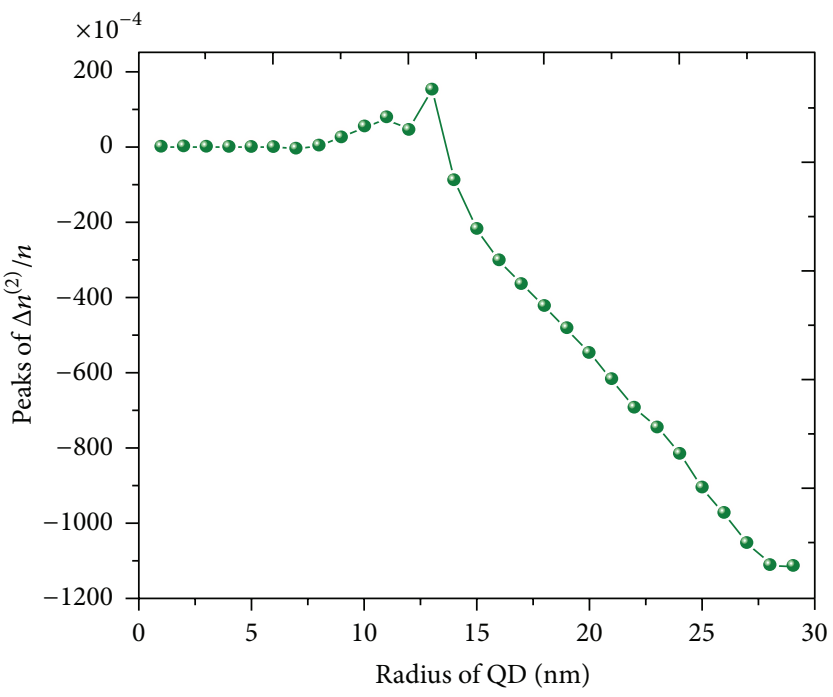

(a)

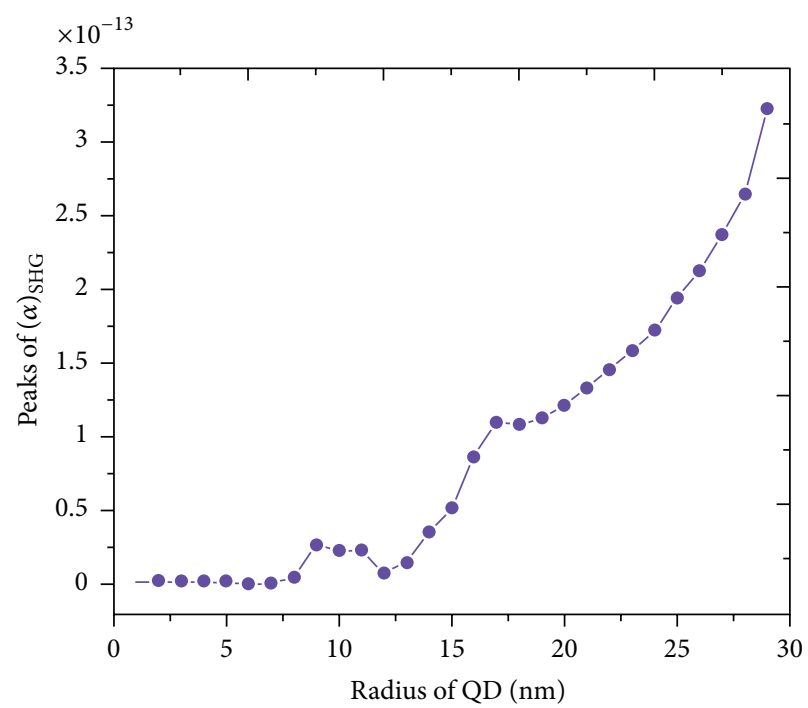

(b)

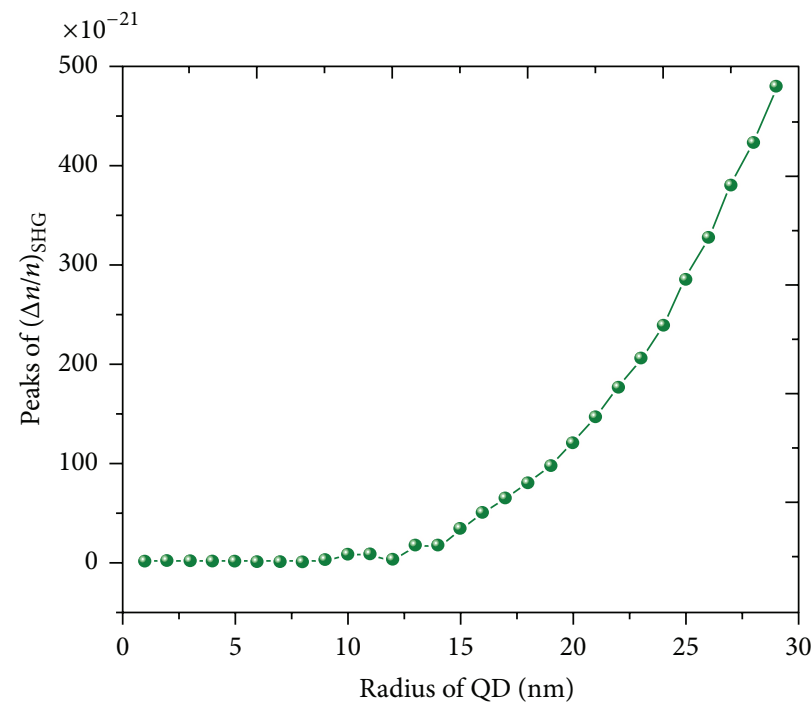

(c)

Figure 7: The biggest peaks of (a) refractive index changes due to optical rectification, (b) absorption due to second harmonic generation, and (c) refractive index changes due to second harmonic generation.

next one just begins. In the dimensions which the envelop function is part in/part out the dipole momentums take close value with almost zero slope. When the FES envelop function becomes localized inside the dot completely at $R=13 \mathrm{~nm}$ it looks like the $z$ component takes the control and the absolute amplitude just keeps rising in the quasi-linear sequence.

For $M_{33}\left(M_{33}=\left\langle\psi_{3}|-e z| \psi_{3}\right\rangle\right)$, the situation is same with somehow more complexities since the SES consists of 3 lobes. Again like the other discussed parameters, the linear increasing behavior shows up on $R=16 \mathrm{~nm}$.

Optical rectification is purely real so according to (9) it only contributes to refractive index changes. Figure 4 shows the mutual refractive changes versus the field frequency for different sizes. Figure 4(a) shows that the significant changes on the OR coefficient begin to appear at $R=5 \mathrm{~nm}$ in which right peaks take smaller values. At $R=6 \mathrm{~nm}$ changes become more significant in which the positive right peaks take negative values and also the left peaks take smaller value. Figure 4(b) shows that in $R=7 \mathrm{~nm}$ the negative take approximately 5 times larger value with redshift and the left peaks become negative. In $R=8 \mathrm{~nm}$ suddenly the right peak faces new exchange and both peaks turn to become positive. The sudden change is mutual to the entrance of GS to dot completely. The same profile is repeated for upper radii with blue shift as well as for the height of the peaks until $R=11 \mathrm{~nm}$ as Figure 4(c) depicts. According to Figure 4(d) at $R=12 \mathrm{~nm}$ suddenly a left peak rises and takes the higher value than the previous radius. At $R=13 \mathrm{~nm}$ two peaks take close values 
and get closer to each other. At $R=14 \mathrm{~nm}$ the right peaks turn to become negative but the left peaks remain positive with smaller absolute value. For upper radii the same profile repeats itself but the absolute amplitude of the peaks rises; for instance, in $R=28 \mathrm{~nm}$ as shown in Figure 4(e) it get the value almost equal to 10 times larger than the absolute peaks of $R=14 \mathrm{~nm}$.

Figure 5 represents the absorption due to SHG. According to Figure 5(a) changes appear on $R=5 \mathrm{~nm}$ and become remarkable on $R=6 \mathrm{~nm}$ where the absolute value of the peaks vanished to relatively small value. At $R=$ $7 \mathrm{~nm}$ the left peaks as well as the right peaks take higher negative amplitudes; mainly only amplification shows up as Figure 5(b) illustrates. At $R=8 \mathrm{~nm}$ the left peaks take smaller absolute values but in positive region also the right peaks turn to become negative. $R=7 \mathrm{~nm}$ and $8 \mathrm{~nm}$ reveal a vice versa profile. At $R=9 \mathrm{~nm}$ everything changes; double positive peaks appear on the right side alongside with other negative peaks on left side as Figure 5(c) depicts. According to Figure 5(d) in the couple of next steps for $R=10$ and $11 \mathrm{~nm}$ the situation is almost the same for the two positive peaks on right side and two negative in left side. On the other hand the peaks have relatively close absolute values. $R=12 \mathrm{~nm}$ shows a reduction on the values as well as the entire change on the profile, in which one peak and valley occur on the right side and the left peaks become positive with so slight relative value. $R=13 \mathrm{~nm}$ according to Figure 5(e) looks so weird since suddenly the right peak vanished and a big valleys takes place with two other right peaks. At $R=14 \mathrm{~nm}$ the two successive valley on the left side of the graph take place while only one positive peak shows up on right side. The sequence keeps repeating itself with intensifying the values of the peaks versus the upper radii according to Figure 5(e).

Figure 6 shows the refractive index changes due to SHG. Just like $\alpha_{\mathrm{SHG}}$ the remarkable impact of size promotion appears on $R=6 \mathrm{~nm}$ as Figure 6(a) illustrates. For $R=7,8$, and $9 \mathrm{~nm}$ in Figures 6(b), 6(c), and 6(d) the refractive index changes (RFC) take a chaotic successive peaks and valleys and the values for these three mentioned radii take extraordinary variations. At $R=10$ and $11 \mathrm{~nm}$ (Figure 6(e)) the RFC graphs take a regular behavior for these two steps with blue shift. Figure 6(d) shows the RFC for $R=12$ and $13 \mathrm{~nm}$. The irregular result takes place for $12 \mathrm{~nm}$ without agreement with the preceding dimensions. $R=13 \mathrm{~nm}$ is the beginning of new behavior for overall shape of RFC curves. In all upper researched radii (Figures 6(e) and 6(f)) 3 successive peaks and valleys are available. The second and third peaks-valley come closer to each other in the upper radii. On the other hand the absolute values of the biggest peaks face incredible intensification.

Figure 7 shows the biggest peak value for refractive index changes because of optical rectification (Figure 7(a)), for the absorption coefficient because of SHG (Figure 7(b)), and the refractive index changes owing to SHG (Figure $7(\mathrm{c})$ ), respectively. The amplitude of the peaks is almost constant comparatively until $R=9 \mathrm{~nm}$. For upper radii the changes in the absolute value of the peaks somehow take hundred time larger values than those of smaller radii.

\section{Conclusion}

In this work the effect of size on transition frequencies, all elements of dipole momentum matrix, ORs and SHG are investigated deeply. All resonant and none-resonant expressions of OR and SHG are taken into consideration, which makes the result more useful for practical applications. The WL caused weird behavior in all discussed parameters. The transition frequencies between subbands satisfy the general expectation, which quotes $\omega_{13} \gg \omega_{12} \gg \omega_{23}$.

At $R=13 \mathrm{~nm}$ in does not face same circumstance on difference transition and in every subband it is the beginning for new sequence. For the absorption and refractive index changes due to optical rectification and SHG, $R=13 \mathrm{~nm}$ is always turning point. As inference it could be concluded that in QDs system with WL there is always critical radius after which the result takes regular behavior sequence. The order of the SHG both in real and imaginary part is relatively smaller than OR by almost the order of $10^{-16}$. The presented result quotes that, in InAs/GaAs QDs, between the second order nonlinear phenomena, the OR plays a dominant role, moreover it is possible to engineer the amplitudes by choosing proper and optimum size for QD.

\section{References}

[1] C. Bose, C. Chakraborty, and C. K. Sarkar, "Electric field induced shifts of electronic energy levels in spherical quantum dot," Solid-State Electronics, vol. 41, no. 9, pp. 1383-1385, 1997.

[2] L. Jacak, P. Hawrylak, and A. Wojs, Quantum Dots, Springer, Berlin, Germany, 1998.

[3] A. M. Elabsy, "Effect of temperature on the binding energy of a confined impurity to a spherical semiconductor quantum dot," Physica Scripta, vol. 59, no. 4, pp. 328-330, 1999.

[4] C. Bose, "Perturbation calculation of impurity states in spherical quantum dots with parabolic confinement," Physica E, vol. 4, no. 3, pp. 180-184, 1999.

[5] V. L. Nguyen, M. T. Nguyen, and T. D. Nguyen, "Magnetic field effects on the binding energy of hydrogen impurities in quantum dots with parabolic confinements," Physica B, vol. 292, no. 1-2, pp. 153-159, 2000.

[6] A. D. Yoffe, "Semiconductor quantum dots and related systems: electronic, optical, luminescence and related properties of low dimensional systems," Advances in Physics, vol. 50, no. 1, pp. 1208, 2001.

[7] E. C. Niculescu, "Energy spectra of donors in spherical quantum dots with parabolic confinement," Czechoslovak Journal of Physics, vol. 51, no. 11, pp. 1205-1213, 2001.

[8] Y. Masumoto and T. Takagahara, Semiconductor Quantum Dots, Springer, Berlin, Germany, 2002.

[9] S. Sahoo, Y. C. Lin, and Y. K. Ho, "Quantum-confined hydrogenic impurity in a spherical quantum dot under the influence of parallel electric and magnetic fields," Physica E, vol. 40, no. 10, pp. 3107-3114, 2008.

[10] I. Karabulut, H. Şafak, and M. Tomak, "Excitonic effects on the nonlinear optical properties of small quantum dots," Journal of Physics D, vol. 41, no. 15, Article ID 155104, 2008.

[11] L. He and W. Xie, "Effects of an electric field on the confined hydrogen impurity states in a spherical parabolic quantum dot," Superlattices and Microstructures, vol. 47, no. 2, pp. 266-273, 2010. 
[12] S. J. Liang and W. F. Xie, "The hydrostatic pressure and temperature effects on a hydrogenic impurity in a spherical quantum dot," The European Physical Journal B, vol. 81, no. 1, pp. 79-84, 2011.

[13] W. Xie and Q. Xie, "Electric field effects of hydrogenic impurity states in a disc-like quantum dot," Physica B, vol. 404, no. 12-13, pp. 1625-1628, 2009.

[14] K. Nishi, T. Kageyama, M. Yamaguchi et al., "Molecular beam epitaxial growths of high-optical-gain InAs quantum dots on GaAs for long-wavelength emission," Journal of Crystal Growth, vol. 378, pp. 459-462, 2013.

[15] T. Sugaya, R. Oshima, K. Matsubara, and S. Niki, "In(Ga)As quantum dots on In GaP layers grown by solid-source molecular beam epitaxy," Journal of Crystal Growth, vol. 378, pp. 430-434, 2013.

[16] T. Li, Q. Wang, X. Guo et al., "The saturation density property of (B)InAs/GaAs quantum dots grown by metal-organic chemical vapor deposition," Physica E, vol. 44, no. 7-8, pp. 1146-1151, 2012.

[17] C. Himwas, R. Songmuang, L. S. Dang et al., "Thermal stability of the deep ultraviolet emission from AlGaN/AlN StranskiKrastanov quantum dots," Applied Physics Letters, vol. 101, no. 24, Article ID 241914, 2012.

[18] H. Hassanabadi, G. Liu, and L. Lu, "Nonlinear optical rectification and the second-harmonic generation in semi-parabolic and semi-inverse squared quantum wells," Solid State Communications, vol. 152, no. 18, pp. 1761-1766, 2012.

[19] M. Barati, G. Rezaei, and M. R. K. Vahdani, "Binding energy of a hydrogenic donor impurity in an ellipsoidal finite-potential quantum dot," Physica Status Solidi B, vol. 244, no. 7, pp. 26052610, 2007.

[20] K. J. Kuhn, G. U. Lyengar, and S. Yee, "Free carrier induced changes in the absorption and refractive index for intersubband optical transitions in AlxGal-xAs/GaAs/AlxGal-xAs quantum wells," Journal of Applied Physics, vol. 70, article 5010, 1991.

[21] W.Xie, "The nonlinear optical rectification of a confined exciton in a quantum dot," Journal of Luminescence, vol. 131, no. 5, pp. 943-946, 2011.

[22] M. Bass, P. A. Franken, J. F. Ward, and G. Weinreich, "Optical rectification," Physical Review Letters, vol. 9, no. 11, pp. 446-448, 1962.

[23] P. A. Franken, A. E. Hill, C. W. Peters, and G. Weinreich, "Generation of optical harmonics," Physical Review Letters, vol. 7, no. 4, pp. 118-119, 1961.

[24] R. W. Boyd, Nonlinear Optics, Elsevier, 3rd edition, 2008.

[25] S. L. Chuang, S. Schmitt-Rink, B. I. Greene, P. N. Saeta, and A. F. J. Levi, "Optical rectification at semiconductor surfaces," Physical Review Letters, vol. 68, no. 1, pp. 102-105, 1992.

[26] K.-X. Guo and S.-W. Gu, "Nonlinear optical rectification in parabolic quantum wells with an applied electric field," Physical Review B, vol. 47, no. 24, pp. 16322-16325, 1993.

[27] O. A. Aktsipetrov, P. V. Elyutin, A. A. Nikulin, and E. A. Ostrovskaya, "Size effects in optical second-harmonic generation by metallic nanocrystals and semiconductor quantum dots: the role of quantum chaotic dynamics," Physical Review B, vol. 51, no. 24, pp. 17591-17599, 1995.

[28] Z.-H. Zhang, K.-X. Guo, B. Chen, R.-Z. Wang, and M.-W. Kang, "Nonlinear optical rectification in cubical quantum dots," Physica B, vol. 404, no. 16, pp. 2332-2335, 2009.

[29] W. Xie, "Nonlinear optical rectification of a hydrogenic impurity in a disc-like quantum dot," Physica $B$, vol. 404, no. 21, pp. 4142-4145, 2009.
[30] C. M. Duque, M. E. Mora-Ramos, and C. A. Duque, "Hydrostatic pressure and electric field effects and nonlinear optical rectification of confined excitons in spherical quantum dots," Superlattices and Microstructures, vol. 49, no. 3, pp. 264-268, 2011.

[31] S. Baskoutas, E. Paspalakis, and A. F. Terzis, "Effects of excitons in nonlinear optical rectification in semiparabolic quantum dots," Physical Review B, vol. 74, no. 15, Article ID 153306, 2006.

[32] C. M. Duque, M. E. Mora-Ramos, and C. A. Duque, "Effects of hydrostatic pressure and electric field on the nonlinear optical rectification of strongly confined electronhole pairs in GaAs quantum dots," Physica E, vol. 43, no. 4, pp. 1002-1006, 2011.

[33] T. Chen, W. Xie, and S. Liang, "The nonlinear optical rectification of an ellipsoidal quantum dot with impurity in the presence of an electric field," Physica E, vol. 44, no. 4, pp. 786-790, 2012.

[34] B. Vaseghi, G. Rezaei, V. Azizi, and S. M. Azami, "Spinorbit interaction effects on the optical rectification of a cubic quantum dot," Physica E, vol. 44, no. 7-8, pp. 1241-1243, 2012.

[35] L. Bouzaïene, R. B. Mahrsia, M. Baira, L. Sfaxi, and H. Maaref, "Hydrostatic pressure and temperature effects on nonlinear optical rectification in a lens shape InAs/GaAs quantum dot," Journal of Luminescence, vol. 135, pp. 271-275, 2013.

[36] G. Liu, K. Guo, Q. Wua, and J. H. Wub, "Polaron effects on the optical rectification and the second harmonic generation in cylindrical quantum dots with magnetic field," Superlattices and Microstructures, vol. 53, pp. 173-183, 2013.

[37] T. Brunhes, P. Boucaud, S. Sauvage et al., "Midinfrared secondharmonic generation in p-type InAs/GaAs self-assembled quantum dots," Applied Physics Letters, vol. 75, no. 6, pp. 835$837,1999$.

[38] A. Liu and G. W. Bryant, "Near-field second-harmonic generation of semiconductor quantum dots," Physical Review B, vol. 59, no. 3, pp. 2245-2253, 1999.

[39] T. Brunhesa, P. Boucauda, S. Sauvagea et al., "Second-harmonic generation in InAs/GaAs self-assembled quantum dots," Physica E, vol. 7, pp. 155-158, 2000.

[40] T. Brunhesa, P. Boucauda, S. Sauvagea et al., "Infrared secondorder optical susceptibility in InAs/GaAs self-assembled quantum dots," Physical Review B, vol. 61, no. 8, pp. 5562-5570, 2000.

[41] T. Brunhesa, P. Boucauda, S. Sauvagea et al., "Second-harmonic generation resonant with s-p transition in InAs/GaAs selfassembled quantum dots," Physical Review B, vol. 63, no. 11, Article ID 113312, 4 pages, 2001.

[42] B. Li, K.-X. Guo, C.-J. Zhang, and Y.-B. Zheng, "The secondharmonic generation in parabolic quantum dots in the presence of electric and magnetic fields," Physics Letters A, vol. 367, no. 6, pp. 493-497, 2007.

[43] S. Shao, K.-X. Guo, Z.-H. Zhang, N. Li, and C. Peng, "Studies on the second-harmonic generations in cubical quantum dots with applied electric field," Physica B, vol. 406, no. 3, pp. 393396, 2011.

[44] M. Sabaeian and A. Khaledi-Nasab, "Size-dependent intersubband optical properties of dome-shaped InAs/GaAs quantum dots with wetting layer," Applied Optics, vol. 51, no. 18, pp. 41764185, 2012.

[45] C. Sun, P. Lu, Z. Yu, H. Cao, and L. Zhang, "Wetting layers effect on InAs/GaAs quantum dots," Physica B, vol. 407, no. 22, pp. 4440-4445, 2012.

[46] T. Konishi and S. Tsukamoto, "Spatial point analysis of quantum dot nucleation sites on InAs wetting layer," Surface Science, vol. 605, no. 5-6, pp. L1-L5, 2011. 
[47] F. Adler, M. Geiger, A. Bauknecht et al., "Optical transitions and carrier relaxation in self assembled InAs/GaAs quantum dots," Journal of Applied Physics, vol. 80, no. 7, pp. 4019-4026, 1996.

[48] O. Stier, M. Grundmann, D. Bimberg et al., "Electronic and optical properties of strained quantum dots modeled by 8-band k.p theory," Physical Review B, vol. 59, no. 8, pp. 5688-5701, 1999.

[49] Y. Li, O. Voskoboynikov, C. P. Lee, and S. M. Sze, "Computer simulation of electron energy levels for different shape InAs/GaAs semiconductor quantum dots," Computer Physics Communications, vol. 141, no. 1, pp. 66-72, 2001.

[50] R. V. N. Melnik and M. Willatzen, "Modelling coupled motion of electrons in quantum dots with wetting layers," in Proceedings of the International Conference on Modeling and Simulation of Microsystems (MSM '02), pp. 506-509, San Juan, Puerto Rico, USA, April 2002.

[51] R. Oshima, N. Kurihara, H. Shigekawa, and Y. Okada, "Electronic states of self-organized InGaAs quantum dots on GaAs (3 11 1) B studied by conductive scanning probe microscope," Physica E, vol. 21, no. 2-4, pp. 414-418, 2004.

[52] R. V. N. Melnik and K. N. Zotsenko, "Finite element analysis of coupled electronic states in quantum dot nanostructures," Modelling and Simulation in Materials Science and Engineering, vol. 12, no. 3, pp. 465-477, 2004.

[53] F. Zhang, L. Zhang, Y.-X. Wang, and R. Claus, "Enhanced absorption and electro-optic Pockels effect of electrostatically self-assembled CdSe quantum dots," Applied Optics, vol. 44, no. 19, pp. 3969-3976, 2005.

[54] A. D. Seddik and I. Zorkani, "Optical properties of a magnetodonor in a quantum dot," Physica E, vol. 28, no. 4, pp. 339-346, 2005.

[55] X.-F. Yang, X.-S. Chen, W. Lu, and Y. Fu, "Effects of shape and strain distribution of quantum dots on optical transition in the quantum dot infrared photodetectors," Nanoscale Research Letters, vol. 3, no. 12, pp. 534-539, 2008.

[56] A. Rostami, H. R. Saghai, N. Sadoogi, and H. B. A. Nejad, "Proposal for ultra-high performance infrared quantum dot," Optics Express, vol. 16, no. 4, pp. 2752-2763, 2008.

[57] C.-H. Liu and B.-R. Xu, "Theoretical study of the optical absorption and refraction index change in a cylindrical quantum dot," Physics Letters A, vol. 372, no. 6, pp. 888-892, 2008.

[58] M. R. K. Vahdani and G. Rezaei, "Intersubband optical absorption coefficients and refractive index changes in a parabolic cylinder quantum dot," Physics Letters A, vol. 374, no. 4, pp. 637$643,2010$.

[59] W. Xie, "Laser radiation effects on optical absorptions and refractive index in a quantum dot," Optics Communications, vol. 283, no. 19, pp. 3703-3706, 2010.

[60] G. Rezaei, Z. Mousazadeh, and B. Vaseghi, "Nonlinear optical properties of a two-dimensional elliptic quantum dot," Physica E, vol. 42, no. 5, pp. 1477-1481, 2010.

[61] L. Lu and W. Xie, "Impurity and exciton effects on the nonlinear optical properties of a disc-like quantum dot under a magnetic field," Superlattices and Microstructures, vol. 50, no. 1, pp. 40-49, 2011.

[62] W. Xie, "A study of nonlinear optical properties of a negative donor quantum dot," Optics Communications, vol. 284, no. 19, pp. 4756-4760, 2011.

[63] S. Liang and W. Xie, "Effects of the hydrostatic pressure and temperature on optical properties of a hydrogenic impurity in the disc-shaped quantum dot," Physica B, vol. 406, no. 11, pp. 2224-2230, 2011.
[64] R. V. N. Melnik and K. N. Zotsenko, "Finite element analysis of coupled electronic states in quantum dot nanostructures," Modelling and Simulation in Materials Science and Engineering, vol. 12, no. 3, pp. 465-477, 2004.

[65] T. Walther, A. G. Cullis, D. J. Norris, and M. Hopkinson, "Nature of the Stranski-Krastanow transition during epitaxy of InGaAs on GaAs," Physical Review Letters, vol. 86, no. 11, pp. 2381-2384, 2001.

[66] J. J. Coleman, J. D. Young, and A. Garg, "Semiconductor quantum dot lasers: a tutorial," Journal of Lightwave Technology, vol. 29, no. 4, Article ID 5664751, pp. 499-510, 2011.

[67] P. A. S. Jorge, M. Mayeh, R. Benrashid, P. Caldas, J. L. Santos, and F. Farahi, "Applications of quantum dots in optical fiber luminescent oxygen sensors," Applied Optics, vol. 45, no. 16, pp. 3760-3767, 2006.

[68] A. Luque, A. Marti, E. Antolin, and P. Garcia-Linares, "Intraband absorption for normal illumination in quantum dot intermediate band solar cells," Solar Energy Materials \& Solar Cells, vol. 94, no. 12, pp. 2032-2035, 2010.

[69] Y.-K. Ee, H. Zhao, R. A. Arif, M. Jamil, and N. Tansu, "Selfassembled InGaN quantum dots on GaN emitting at $520 \mathrm{~nm}$ grown by metalorganic vapor-phase epitaxy," Journal of Crystal Growth, vol. 310, no. 7-9, pp. 2320-2325, 2008. 

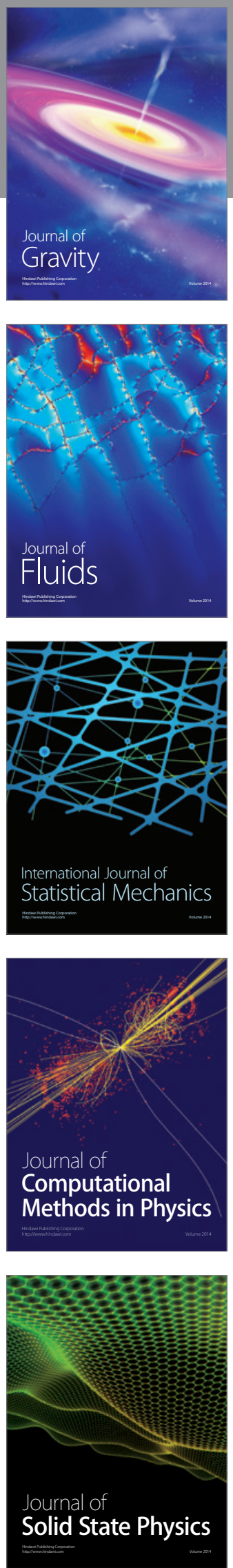

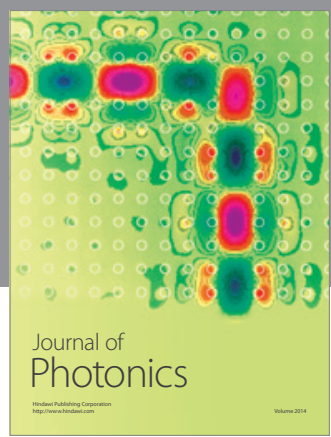

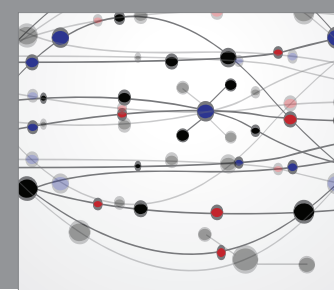

The Scientific World Journal

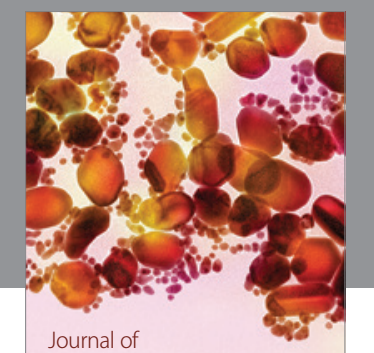

Soft Matter
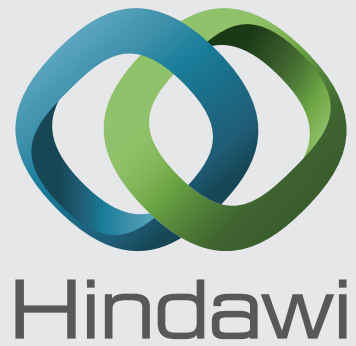

Submit your manuscripts at

http://www.hindawi.com
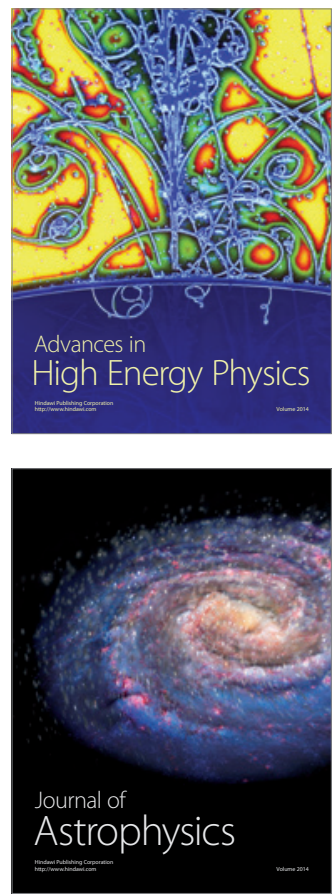
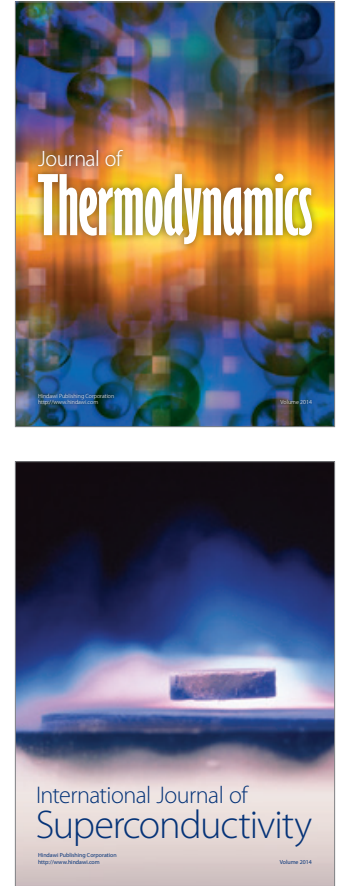
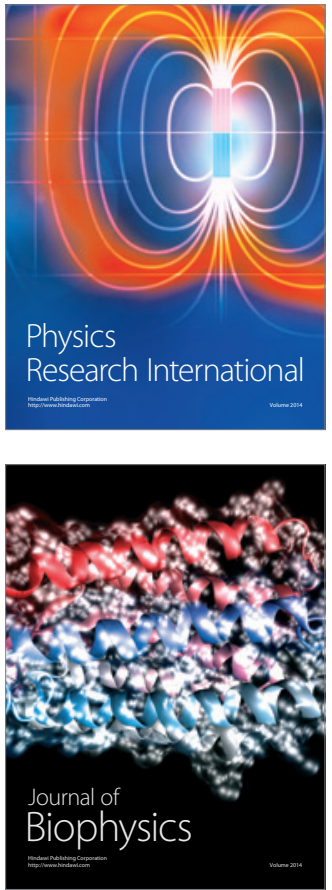
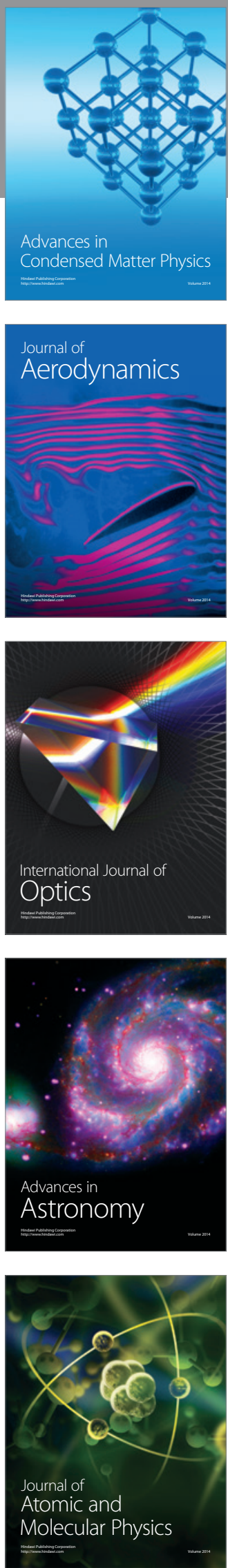